\title{
OSTEOMYELITIS OF THE CALCANEUM
}

\author{
E. H. M. WANG, S. SIMPSON, GEORGE C. BENNET
}

From the Royal Hospital for Sick Children, Glasgow

\begin{abstract}
We reviewed 52 cases of osteomyelitis of the calcaneum. The clinical symptoms and signs were well defined, but different and less dramatic than those of long-bone osteomyelitis. Blood cultures were positive in $41 \%$ of cases and tissue cultures in $91 \%$. Routine haematological tests were of little value, and radiological changes were often delayed, and were absent in $12 \%$.

With early diagnosis, treatment with antibiotics alone was usually effective, but complications and chronic disease were more likely if there was delay. Early diagnosis is the key to successful treatment. We describe a new physical sign and consider that diagnosis is almost always possible by clinical methods.
\end{abstract}

Osteomyelitis of the long bones is the subject of many papers and its classical presentation is well known. The diagnosis is seldom missed and early treatment usually gives good results. Less attention has been paid to the diagnosis and management of bacterial infection of short bones, which is less common, but not rare, comprising about $28 \%$ of all osteomyelitis (Feigin et al 1970 ).

The calcaneum is involved in $7 \%$ to $8 \%$ of all cases of osteomyelitis (Feigin et al 1970; Antoniou and Conner 1974), but no large series has been reported. Most accounts are either case reports or part of a larger series describing bone infection at unusual sites (Brand and Black 1974; Morrey, Bianco and Rhodes 1978).

We have reviewed the causes, clinical presentation and outcome of calcaneal osteomyelitis at our hospital, with emphasis on any differences from the better-known long-bone disease.

\section{PATIENTS AND METHODS}

We made a retrospective study of all cases of calcaneal osteomyelitis seen at a specialist children's hospital from 1970 to 1990 . The criteria for inclusion in the study were localisation of signs or symptoms to the heel, and at least one of the following: a positive blood culture, a positive

E. H. M. Wang, MD, Consultant

Department of Orthopaedics, University of the Philippines, Philippine General Hospital, Manila, Republic of the Philippines.

S. Simpson, FRCS, Senior Orthopaedic Registrar

Dundee Royal Infirmary, Barrack Road, Dundee DD1 9ND, Scotland.

G. C. Bennet, FRCS, Consultant Orthopaedic Surgeon

Department of Orthopaedics, Royal Hospital for Sick Children, Yorkhill, Glasgow G3 8SJ, Scotland.

Correspondence should be sent to Mr G. C. Bennet.

(C) 1992 British Editorial Society of Bone and Joint Surgery

0301-620X/92/6467\$2.00

J Bone Joint Surg [ Br] 1992; 74-B:906-9.
Table I. The ages of 52 children at time of diagnosis of calcaneal osteomyelitis

\begin{tabular}{lc}
\hline Age (years) & Number \\
\hline 0 to 1 & 2 \\
1 to 5 & 14 \\
5 to 10 & 19 \\
10 to 14 & 17 \\
\hline
\end{tabular}

wound culture, typical radiographic changes, or a positive bone scan.

The case records and radiographs of 52 patients who qualified for inclusion were reviewed. The average followup was 11 months (three months to three years). Five patients had been lost to follow-up before discharge from the clinic, but four of these were symptom-free at their latest review. The fifth still had some pain when last seen, but radiographs taken at that stage showed signs of healing.

\section{RESULTS}

There were 26 boys and 26 girls; their ages are given in Table I. The right heel was affected in 28 and the left in 24. Of the two below the age of one year, one was diagnosed at 19 days of age and the other at two months. Thirteen children had a history of trauma, but only four had suffered from a puncture wound. We could find no evidence of any relationship to trauma in any of the other 39 patients.

Clinical findings. The clinical presentation was remarkably consistent. Forty-five patients $(90 \%$ of those over one year of age), had a limp or refused to bear weight and $45(86 \%$ of all patients) showed local inflammation. Only 
one-third had systemic symptoms with pyrexia. In all cases there was point tenderness around the heel, but despite the essential search, a puncture wound was rarely found (Fig. 1).

A number of our recent patients have shown a characteristic posture: they did not allow the heel of the affected limb to come into contact with the bed, even when they were asleep. We termed this the 'heel-up' sign (Fig. 2). We did not see it in children with heel pain caused by pathology other than infection, or in cases of osteomyelitis of other tarsal bones. It appears that this sign may be diagnostic.

Duration of symptoms. Despite the characteristic clinical picture, there was frequently a delay in diagnosis, exceeding five days in 17 patients and two weeks in eight (Table II). The reasons for delay and the mistaken diagnoses are given in Table III. Delay before admission, in 11 children seen by a family practitioner or a casualty officer, was most commonly because infection was not suspected. After admission, localisation was more com-

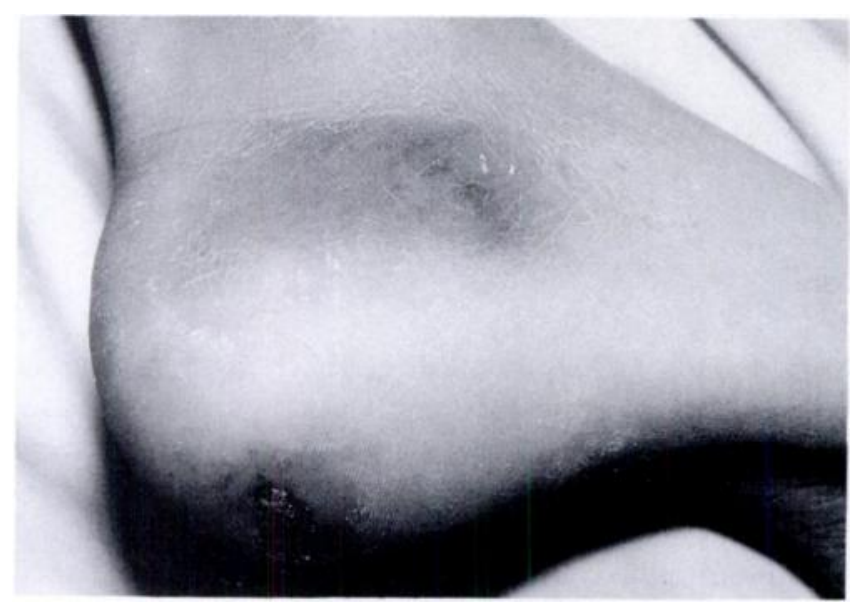

Fig. 1

Puncture wound of the heel with signs of localised inflammation. Pseudomonas aeruginosa was isolated.

monly the problem. In two children, there were delays in diagnosis both before and after admission. The four children with puncture wounds were all diagnosed within four days.

Laboratory investigations. The earliest white cell count, performed in 47 patients, was over $14000 / \mu l$ in only six $(13 \%)$. There was elevation of the erythrocyte sedimentation rate (ESR) in 38 of 48 children ( $79 \%$ ) but rarely to a degree that aided diagnosis. In only one case did the ESR exceed $100 \mathrm{~mm}$ in the first hour (Table IV).

Blood culture had been performed in 37 cases, and $41 \%$ were positive. Cultures taken at operation were positive in 20 of 22 cases $(91 \%)$. The organisms are given in Table $\mathrm{V}$. We excluded four patients with negative cultures taken by incorrect procedures, such as by ankle aspiration. Taking both investigations, a positive culture was obtained in 28 of the 52 patients; all four children with puncture wounds were infected with Pseudomonas aeruginosa.

Radiography. At first presentation, 20 patients had radiographic changes and by three weeks these were present in 35 of the 52 cases $(67 \%)$. Six patients $(12 \%)$ never showed any radiographic changes.

The usual abnormality was resorption (Fig. 3); periosteal elevation was seen in only two cases. Changes were most common in the posterior or 'metaphyseal equivalent' area of the calcaneum (Nixon 1978), with the posterior third of the bone involved in $76 \%$.

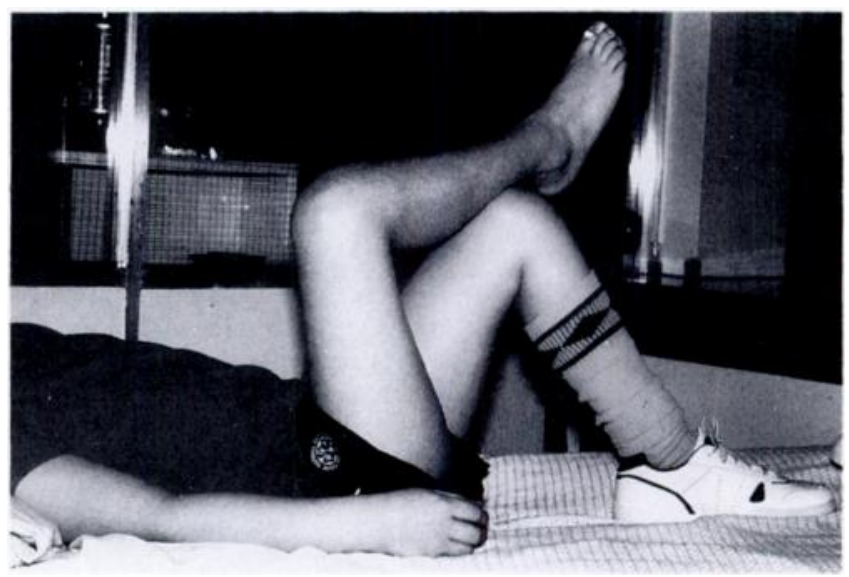

Fig. 2

The 'heel-up' sign

Table II. Delay in treatment before admission to hospital

\begin{tabular}{ll}
\hline Delay (days) & Number \\
\hline$<2$ & 17 \\
2 to 5 & 18 \\
5 to 14 & 9 \\
$>14$ & 8 \\
\hline
\end{tabular}

Table III. Misdiagnosis causing delay before and after admission in 52 children with calcaneal osteomyelitis

\begin{tabular}{ll}
\hline Misdiagnosis & Number \\
\hline Before admission & \\
Normal & 5 \\
Contusion & 2 \\
Sprain & 1 \\
Soft-tissue abscess & 1 \\
Sever's disease & 1 \\
Irritable hip & 1 \\
& \\
After admission & \\
Septic arthritis & 6 \\
Soft-tissue abscess & 4 \\
Calcaneal tendonitis & 2 \\
Irritable hip or early Perthes' & 2 \\
disease & \\
Osteomyelitis of the tibia & 1 \\
Cellulitis & 1 \\
\hline
\end{tabular}




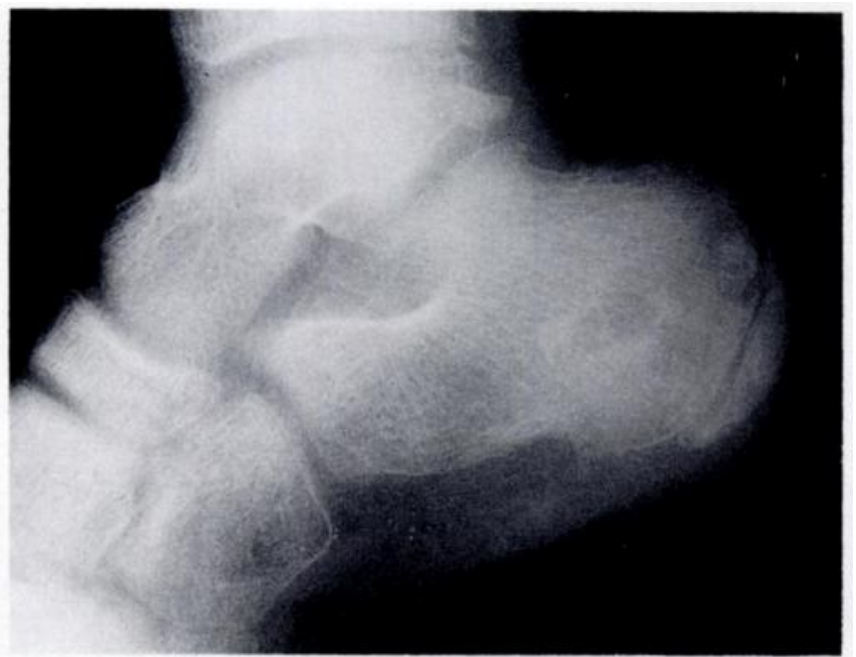

Fig. 3

Typical radiographic appearance showing erosive changes in the posterior part of the bone.

Table IV. Results of blood tests in children with calcaneal osteomyelitis

\begin{tabular}{lll}
\hline Blood test & Number & Per cent \\
\hline Initial ESR & & \\
$<20 \mathrm{~mm} / \mathrm{hr}$ & 10 & 21 \\
$>20 \mathrm{~mm} / \mathrm{hr}$ & 38 & 79 \\
\multicolumn{2}{l}{ Initial white cell count } \\
$<10000 / \mu \mathrm{l}$ & 20 & 43 \\
$>10000 / \mu \mathrm{l}$ & 27 & 57 \\
$>14000 / \mu \mathrm{l}$ & 6 & 13 \\
\hline
\end{tabular}

Table V. Organisms cultured at operation in 22 children with calcaneal osteomyelitis

\begin{tabular}{lc}
\hline Organism & Number \\
\hline $\begin{array}{l}\text { Coagulase-negative } \\
\text { Staphylococcus aureus }\end{array}$ & 1 \\
Coagulase-positive & \\
$\quad$ Staphylococcus aureus & 11 \\
Pseudomonas aeruginosa & $4^{*}$ \\
Streptococcus pyogenes & 2 \\
Gram-positive cocci & 2 \\
Sterile & 2 \\
\hline
\end{tabular}

* all had puncture wounds

Bone scanning was most useful when there was a short history of symptoms and the plain films were normal. Positive features were an increased uptake on the blood pool scan, and increased focal uptake on the delayed scan (Fig. 4).

\section{Treatment}

Medical. The standard treatment for all the patients that we report was bed-rest, elevation of the leg, posterior splintage and antibiotics prescribed on a 'best guess'

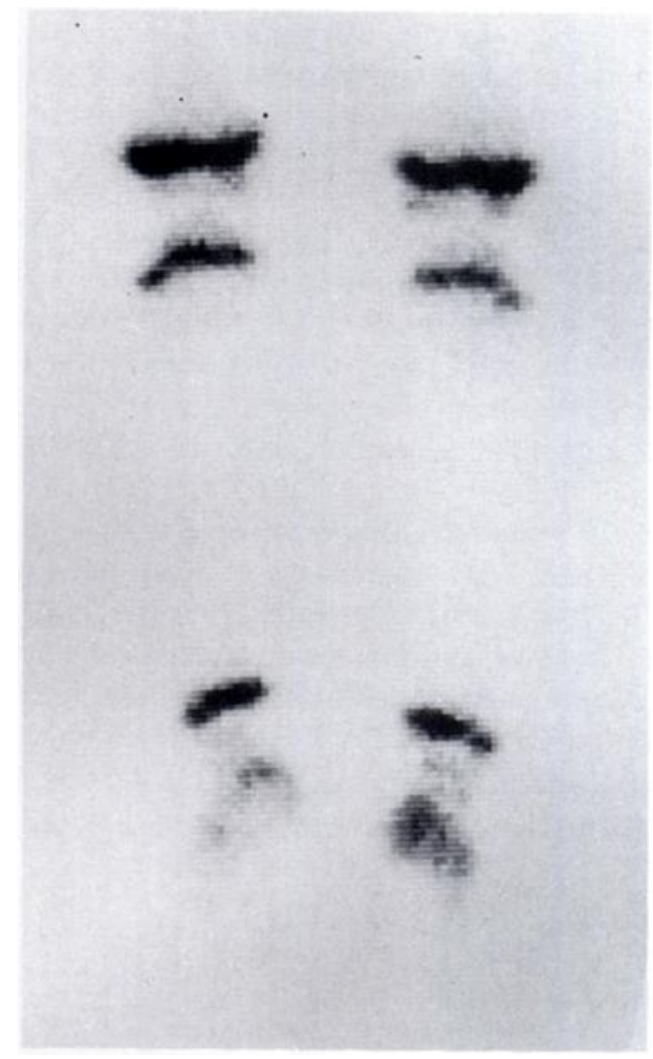

Fig. 4

A bone scan showing the increased uptake in one calcaneum.

policy. Before 1964 , the most generally used antibiotics were penicillin or tetracycline, but when penicillinaseresistant drugs became available, most children were treated with cloxacillin or Fucidin and erythromycin. The standard duration of treatment was three weeks, and this produced 32 uneventful cures $(61 \%)$. The children with pseudomonas infection of a puncture wound were all treated with gentamicin.

Patients were discharged from hospital when clinically improved, whether or not the radiographs showed signs of healing, and the average hospital stay was three weeks (five days to six months). The longest stay was that of a patient who developed extensive complications and had several operations and courses of antibiotics.

Operative. A total of 23 operations were performed; eight of these were because of misdiagnosis. There were six subtalar or ankle aspirations or arthrotomies, and two cases of incision of a soft-tissue abscess. Three of these patients required reoperation for debridement and curettage. The 15 correctly indicated operations were all performed in children who failed to show adequate clinical and radiological response to rest and antibiotics. Complications. As in long-bone infection (Ross and Cole 1985) we found an association between delay in diagnosis and the later development of complications. Eleven patients $(21 \%)$ developed 13 complications, but these were much more common early in the study, with eight 
in 17 cases before 1970 and only three in 35 patients after 1970.

In one patient, infection spread to involve the subtalar joint. Ten developed chronic osteomyelitis, five with sequestrum formation, five without. Two patients had growth abnormalities of the calcaneum secondary to damage to its apophysis.

\section{DISCUSSION}

We believe this to be the largest published series of cases of calcaneal osteomyelitis. Despite the retrospective nature of the study, it revealed a homogeneous group of patients differing in several respects from the betterknown long-bone infection. Two differences were that there was no peak of cases below the age of one, but a fairly even spread throughout childhood, as was also reported by Feigin et al (1970) and Antoniou and Conner (1974), and an equal sex ratio, as against the preponderance of boys over girls in long-bone infection.

Most patients had no definite preceding history of trauma. In many cases an injury was remembered retrospectively in trying to rationalise the symptoms, but such histories are probably not related to the subsequent development of the condition.

Children with puncture wounds tended to present early, often because of the wound itself. This contrasts with the experience of Brand and Black (1974) who found a considerable delay in seeking medical advice in this group. We had no cases secondary to medical heel puncture of neonates although this has been described (Lilien et al 1976).

Local clinical symptoms were common, with a limp or refusal to bear weight, but there was systemic upset in only a few cases. Point tenderness was almost invariable, and the new 'heel-up' sign may prove to be useful as it is highly suggestive of the diagnosis.

As in all bone infections, once the diagnosis is considered, it is unlikely to be missed. It seems surprising that the correct diagnosis is so often delayed, but in the early stages, the symptoms are easily dismissed because of their similarity to the everyday aches and pains of an active child. Medical delay before admission to hospital is usually because infection has not been considered. After admission, infection is usually diagnosed, but its localisation remains a problem. There is little doubt that delay may lead to the development of otherwise a voidable complications such as chronic infection.

We found that laboratory investigations were of limited use, and could be misleading. Elevation of the ESR and white cell count was common, but seldom to a level suggestive of infection. This contrasts with the findings of King and Mayo (1969) who reported that the ESR often exceeded $100 \mathrm{~mm}$ in the first hour in such cases. Blood cultures were positive in slightly less than half those in whom the investigation had been performed, agreeing with the findings of Antoniou and Conner (1974), but in a much higher proportion than that reported by Feigin et al (1970). Direct culture of tissue or pus, however, gave a $91 \%$ positive result.

Radiographic changes were often delayed: only onethird showed any abnormality within two weeks of onset of symptoms. Brand and Black (1974) had a similar experience, finding that changes took between eight and 19 days to become evident. Bone scanning is more likely to produce a positive early result; it is indicated in all suspected cases, both to make the diagnosis and to localise the infection (Howie et al 1983). It may also be of value in a late-presenting case: $12 \%$ of the children in our series never showed any radiographic changes.

In most cases appropriate treatment leads to a satisfactory outcome, with a small number developing complications, such as chronic infection, which require surgery. We found growth disturbance of the calcaneum in two children. This was first reported by Borris and Helleland (1986) who pointed out that the calcaneal apophysis does not ossify until the age of four years, so that such changes may not be radiologically apparent until then.

The lower number of complications in the later years of our study probably reflects an improvement in the use and effectiveness of antibiotics and in the general health of children: delay in diagnosis was not a factor, since this was very similar both before and after 1970 .

No benefits in any form have been received or will be received from a commercial party related directly or indirectly to the subject of this article.

\section{REFERENCES}

Antoniou D, Conner AN. Osteomyelitis of the calcaneus and talus. J Bone Joint Surg [ Am] 1974; 56-A :338-45.

Borris LC, Helleland H. Growth disturbance of the hind part of the foot following osteomyelitis of the calcaneus in the newborn. $J$ Bone Joint Surg [Am] 1986; 68-A :302-5.

Brand R, Black H. Pseudomonas osteomyelitis following puncture wounds in children. J Bone Joint Surg [ Am] 1974; 56-A :1637-42.

Feigin RD, McAlister WH, San Joaqin VH, Middelkamp JN. Osteomyelitis of the calcaneus: report of eight cases. Am J Dis Child 1970; 119:61-5.

Howie DW, Savage JP, Wilson TG, Paterson D. The technetium phosphate bone scan in the diagnosis of osteomyelitis in childhood. J Bone Joint Surg [Am] 1983; 65-A :431-7.

King DM, Mayo KM. Subacute haematogenous osteomyelitis. J Bone Joint Surg [Br] 1969: 51-B:458-63.

Lilien LD, Harris VJ, Ramamurthy RS, Pildes RS. Neonatal osteomyelitis of the calcaneus: a complication of heel puncture. J Pediatr $1976 ; 88: 478-80$.

Morrey BF, Bianco AJ, Rhodes KH. Haematogenous osteomyelitis at uncommon sites in children. Mayo Clin Proc 1978; 53:707-13.

Nixon GW. Haematogenous osteomyelitis of metaphyseal-equivalent locations. AJR 1978; $130: 123-9$.

Ross ERS, Cole WG. Treatment of subacute osteomyelitis in childhood. J Bone Joint Surg [ Br] 1985; 67-B :443-8. 